\title{
LA NECESIDAD DEL CAMBIO EDUCATIVO PARA LA SOCIEDAD DEL CONOCIMIENTO
}

\section{José Ginés Mora $(*)$}

SÍNTESIS: En este artículo se analizan, en primer lugar, los cambios que están teniendo lugar en el contexto de la educación superior, especialmente en la española y en la europea. A continuación, se presentan algunas ideas acerca de cuál debería ser la respuesta de la educación superior frente a estos cambios, con el fin de que las universidades sean capaces de seguir sirviendo a la sociedad, sobre todo a la nueva, que llamamos del conocimiento.

SÍNTESE: Neste artigo, são analisadas, em primeiro lugar, as mudanças que estão acontecendo no contexto da educação superior, especialmente na espanhola e na européia. A seguir, são apresentadas algumas idéias a respeito de qual deveria ser a resposta da educação superior frente a estas mudanças, com o objetivo de que as universidades sejam capazes de seguir servindo à sociedade, especialmente à nova, que chamamos a do conhecimento.

(*) Director del Centro de Estudios en Gestión de la Educación Superior de la Universidad Politécnica de Valencia, España. 


\section{LOS MODELOS UNIVERSITARIOS TRADICIONALES}

Los universitarios tienen cierta tendencia a creer en la inmutabilidad de los principios universitarios. Existe una idea, 0 al menos una sensación compartida, de que los principios que inspiran la organización educativa, el proceso de enseñanza, las relaciones con la investigación, por poner unos ejemplos, han permanecido sin cambios a lo largo de los años, y de que forman parte de las esencias de las instituciones universitarias. Es curioso que los universitarios, muy críticos con la mayoría de los aspectos de la ciencia o de la sociedad, sean tan respetuosos con lo que se llama la cultura universitaria (Sporn, 1996). En consecuencia, las universidades, dirigidas en muchos países por los propios universitarios, se comportan como instituciones conservadoras, sobre todo en los momentos de grandes cambios. Vivimos ahora unos momentos en los que la sociedad está sufriendo mutaciones muy profundas, y sería necesario que la universidad se adaptase a ellas si no quiere verse convertida en una institución obsoleta que ya no responde a las demandas sociales. En este artículo revisaremos algunos de los cambios que están teniendo lugar en la sociedad y que afectan al mundo universitario, para pasar después a proponer al gunas respuestas que ese mundo debería dar a estos cambios de contexto.

Revisaremos, en primer lugar, algunas ideas sobre los modelos históricos de la educación superior. Los universitarios solemos estar muy orgullosos de la vieja y larga vida de las universidades, que se remonta a la Edad Media. Sin embargo, las universidades, tal como hoy las conocemos, son mucho más recientes. Fue a principios del sigloxIX cuando tuvo lugar el gran cambio de la universidad medieval a la universidad moderna. En ese momento aparecieron tres modelos de universidades con organizaciones diferentes, que se corresponden con otras tantas respuestas a la sociedad emergente del siglo XIX. Esta sociedad se caracterizaba por dos hechos: en primer lugar, se trataba de una sociedad en la que adquiría importancia como nuevo modelo de organización social el Estado-nación liberal; en segundo lugar, era la sociedad en la que se estaba produciendo el desarrollo industrial. Ante ese fenómeno común en Europa y en los nuevos Estados americanos: surgimiento de los Estados nacionales y de la era industrial, los países respondieron con diferentes modelos de organización de sus universidades. Dichos modelos se pueden agrupar en tres tipos:

- El alemán, también Ilamado humboldtiano, se organizó mediante instituciones públicas, con profesores funciona- 
rios y con el conocimiento científico como meta de la universidad. En ella, el objetivo era formar personas con amplios conocimientos, no necesariamente relacionadas con las demandas de la sociedad o del mercado laboral. La idea que sustentaba el modelo (heredada del idealismo alemán del siglo XVIII) era que una sociedad con personas formadas científicamente sería capaz de hacer avanzar al conjunto de la sociedad en sus facetas sociales, culturales y económicas. De hecho fue así durante más de un siglo, y las universidades alemanas ayudaron no poco a convertir al país en una potencia científica y económica.

- El modelo francés, también llamado napoleónico, tuvo por objetivo formar a los profesionales que necesitaba el Estadonación burocrático recién organizado por la Francia napoleónica. Las universidades se convirtieron en parte de la administración del Estado para formar a los profesionales que ese mismo Estado necesitaba. Los profesores se harían funcionarios públicos, y las instituciones estarían al servicio del Estado más que al de la sociedad. El modelo, exportado a otros países del sur de Europa, tuvo éxito también para la consolidación de las estructuras del Estado liberal.

- El modelo anglosajón, al contrario de los dos anteriores, no convirtió en estatales a las universidades, manteniendo el estatuto de instituciones privadas que todas las universidades europeas tenían hasta principios del siglo XIX. En las universidades británicas, cuyo modelo se extendió a las norteamericanas, el objetivo central fue la formación de los individuos, con la hipótesis de que personas bien formadas en un sentido amplio serían capaces de servir adecuadamente las necesidades de las nuevas empresas o las del propio Estado. Este modelo, como los otros, también tuvo éxito en los países en los que se aplicó, pero, a diferencia de los otros, resistió mejor el paso del tiempo y parece estar más adaptado al contexto actual.

Los tres modelos de universidades que surgen en los inicios del siglo XIX, han ido entremezclando sus características con el paso del tiempo. Por ejemplo, la investigación científica, una característica típica del modelo alemán a la que eran ajenas las universidades anglosajonas, se incorporó a algunas de ellas a finales del propio sigloxIX. Sin embargo, 
las universidades francesas o algunas otras instituciones de educación superior de ese país, como las Grandes Écoles, siguen siendo ajenas a la idea de que la investigación es una parte esencial de la vida universitaria. Podemos apreciar que al gunas cosas que los universitarios consideramos fundamentales, como la investigación, son ajenas a la universidad antigua, pero también a muchas universidades modernas. Este es un buen ejemplo de que la universidad tiene menos principios sagrados y generales que los que los propios universitarios solemos creer.

España es un caso típico de model o napol eónico de universidad, aunque las reformas que tuvieron lugar durante los años 80 nos separaron algo de ese modelo. Sin embargo, y a pesar de la autonomía y de la formal separación del Estado, las universidades siguen siendo instituciones con un fuerte carácter funcionarial, con un gobierno burocrático, y, sobre todo, con una fuerte orientación profesionalizante (Mora, 2004). Esta última característica de la universidad española, la orientación profesionalizante que compartimos con muchos otros países, especialmente con los latinoamericanos, merece que se le preste especial atención.

El modelo dominante en Latinoamérica se asemeja en lo fundamental al napoleónico, y está concebido para dar respuesta a las necesidades de un mercado laboral caracterizado por:

- Profesiones bien definidas, con escasa intercomunicación, con competencias profesionales claras, y, en muchos casos, hasta legalmente fijadas. La escasa intercomunicación que las profesiones tienen entre ellas, hace que las competencias requeridas sean siempre específicas y relacionadas con un aspecto concreto del mundo laboral.

- Profesiones estables, cuyas exigencias de competencia profesional apenas cambian a lo largo de la vida profesional.

El sistema de educación superior, y de alguna manera el del conjunto del sistema educativo, daba respuesta a estas necesidades específicas del mercado laboral. La palabra «licenciado», de tanto arraigo en nuestros sistemas universitarios, representa bien ese sentido que se le ha dado a la universidad como otorgadora de licencias para ejercer las profesiones. Lógicamente, si se trataba de formar para profesiones que además iban a ser estables durante mucho tiempo, las universidades formaban enseñando el estado del arte en cada profesión. 
Todos los conocimientos que podían ser necesarios para ejercerla debían ser inculcados en los jóvenes estudiantes. La hipótesis era que todo lo que no se aprendía en la universidad ya no se iba a aprender después. Los profesores, actores principales del proceso educativo, debían procurar que los estudiantes aprendieran el máximo de conocimientos específicos que fueran a ser necesarios en la vida laboral, pero, sobre todo, que los profesores deberían garantizar que ningún estudiante que obtuviera el título académico (que igualmente era el profesional) careciera de esos conocimientos imprescindibles para el ejercicio de la profesión. La universidad y el profesor eran -y siguen siendo- garantes de que los graduados tengan la competencia profesional necesaria. Las universidades no sólo dan la habilitación académica sino también la profesional, al contrario de lo que sucede en el mundo anglosajón, en el que la habilitación para el ejercicio profesional la otorgan los gremios profesionales y no las universidades. Este es un hecho relevante que podría cambiar pronto, y que supondría una auténtica revolución en el modelo tradicional de las universidades.

Este modelo educativo, que se creó hace dos siglos, sigue presente en buena medida en la universidad española. Una reciente encuesta (Teichler y Schonburg, 2004) realizada a graduados universitarios europeos y españoles, muestra el parecer que estos graduados tienen sobre el tipo de formación que han recibido en la universidad. La encuesta se realizó en el año 1999 a personas que habían terminado sus estudios cuatro años antes. Por tanto, se trataba de opiniones de graduados que habían asistido a la universidad en la década de los 90 , es decir, durante la época de las reformas educativas que tuvieron lugar a principios de ese período en España. Los resultados de una de las cuestiones planteadas (el énfasis que se hacía en la universidad sobre una serie de aspectos) se muestra en la tabla 1. En la primera columna se presentan los resultados referidos a Europa, y en la segunda los correspondientes a España.

El modelo pedagógico del sistema universitario español queda perfectamente definido en los resultados de esta tabla. Los graduados creen que la universidad hace hincapié en la transmisión de teorías y de conceptos, mientras que el aprendizaje independiente, el conocimiento instrumental, el aprendizaje basado en problemas y en proyectos, las actitudes y habilidades sociales y comunicativas, la adquisición directa de experiencia laboral, no superan el valor central del 5 . Además de teorías y de conceptos, el sistema concede importancia a la asistencia a clase y al consiguiente valor del profesor como fuente fundamental de 
TAB LA 1

Énfasis que se hizo en la universidad en los siguientes aspectos

(Indicador que toma valores entre 0 y 10 )

\begin{tabular}{|l|c|c|}
\hline & Europa & España \\
\hline Teorías y conceptos & 7,6 & 8,1 \\
\hline Asistencia a clase & 5,6 & 6,1 \\
\hline $\begin{array}{l}\text { Importancia del profesor como fuente de } \\
\text { información }\end{array}$ & 5,2 & 6,0 \\
\hline Aprendizaje independiente & 6,5 & 4,5 \\
\hline Hechos y conocimiento instrumental & 6,1 & 4,0 \\
\hline Aprendizaje basado en problemas y proyectos & 4,4 & 3,9 \\
\hline Actitudes y habilidades socio-comunicativas & 4,4 & 3,2 \\
\hline Adquisición directa de experiencia laboral & 3,1 & 1,9 \\
\hline
\end{tabular}

FUENTE: Elaboración propia con datos de la encuesta CHEERS.

información. Podríamos sintetizar la situación, caricaturizándola algo, afirmando que el model o pedagógico de la universidad española consiste ante todo en un profesor contando teorías y conceptos a alumnos sentados con regularidad en el aula. También en Europa (no hay que olvidar que los datos de esta primera columna incluyen a los españoles, a los franceses o a los italianos, en los que también impera el mismo modelo educativo) la teoría y los conceptos son lo más destacado por las universidades, pero, a diferencia de España, el aprendizaje independiente $o$ el aprendizaje de hechos y de conocimientos instrumentales supera el punto central de valoración. Sin llegar al punto medio, pero siempre por encima de la media española, se encuentra el aprendizaje basado en problemas y en proyectos, en el de habilidades sociales y comunicativas, y en la adquisición directa de experiencia laboral. La imagen sobre el model o pedagógico español que ofrecen estos resultados representa bien a las claras un sistema obsoleto, que responde todavía a las necesidades de un mercado laboral decimonónico.

La tabla 2, obtenida de la misma encuesta, confirma esa visión de la formación pedagógica que ofrece la universidad española. Los graduados españoles (como los europeos) valoraban especialmente el 
contacto con los compañeros. También superan el valor central el contenido de los programas o la calidad de la docencia. Sin embargo, el diseño del plan de estudios, es decir, la organización del proceso de enseñanza y aprendizaje, no es aprobado por los graduados españoles, que igualmente valoran de forma negativa el carácter práctico de las enseñanzas, el relieve que se le da a la investigación, o las oportunidades que se les ofrecen para participar en proyectos 0 en prácticas en empresas. Es evidente que el problema de la formación que reciben los universitarios no reside en el contenido de las asignaturas o en la calidad de los profesores, sino en una incorrecta organización del conjunto de los estudios, con muy escaso acento en la práctica o en el proceso de aprendizaje mediante la investigación, la participación en proyectos, las actividades formativas y las prácticas en empresas. Como en la situación anterior, los resultados para el conjunto europeo son mejores que para el caso español en todos los aspectos, aunque también los graduados europeos suspenden a sus universidades en cuanto a la escasa importancia práctica de la enseñanza, a la parca participación en actividades de investigación, y a las exiguas oportunidades para participar en proyectos o en prácticas en empresas.

TABLA 2

Valoración sobre los estudios

(Indicador que toma valores entre 0 y 10 )

\begin{tabular}{|l|c|c}
\hline & Europa & España \\
\hline Contactos con los compañeros & 7,4 & 7,3 \\
\hline Contenido de los programas & 6,2 & 5,5 \\
\hline Calidad de la docencia & 5,7 & 5,0 \\
\hline Diseño del plan de estudios & 5,4 & 4,1 \\
\hline Asesoramiento académico en general & 4,7 & 3,9 \\
\hline Énfasis práctico en la enseñanza & 4,3 & 3,4 \\
\hline Énfasis en la investigación & 3,8 & 2,3 \\
\hline Oportunidades de participar en proyectos & 3,3 & 2,0 \\
\hline Oportunidades para prácticas en empresas & 3,5 & 1,9
\end{tabular}

FUENTE: Elaboración propia con datos de la encuesta CHEERS 


\section{UN NUEVO CONTEXTO PARA LA EDUCACIÓN SUPERIOR}

Como hemos podido apreciar, el modelo de formación universitaria vigente en España, pero que tiene mucho en común con el actual de buena parte de Europa y de Latinoamérica, responde a las necesidades de una sociedad y de un mercado laboral que están desapareciendo. El contexto al que ha de responder la educación superior está cambiando, y es necesario que también se modifique el modelo de formación si se quiere dar respuesta a las necesidades de este nuevo contexto. Revisemos con brevedad cuáles son las características más relevantes de este nuevo contexto para la educación superior.

\subsection{LA SOCIEDAD GLOBAL}

La globalización del contexto de la educación superior es casi una obviedad. El mercado laboral, sobre todo el de los graduados universitarios, se está haciendo global en un doble sentido: no sólo los graduados trabajan con creciente frecuencia en otros países, sino que lo hacen en compañías transnacionales cuyos métodos de trabajo, de organización y de actividades tienen un carácter global. Esa globalización, $y$, por tanto, la de sus requerimientos formativos, afecta de manera muy directa al funcionamiento de las instituciones universitarias, que deberán dar respuesta a unas necesidades de formación que ya no son las específicas de un entorno inmediato.

Otra característica importante de la globalización es la velocidad con la que se mueve el conocimiento. La relativa estabilidad de las profesiones, típica de la era industrial, ligada a unos conocimientos constantes y a un entorno específico, ya no es la situación imperante.

Un tercer aspecto que hay que resaltar de la sociedad global es la competencia global de instituciones de educación superior. Las rondas GATT insisten en la inclusión de la educación superior como un servicio más sometido al libre intercambio que se promueve desde la Organización Mundial del Comercio (Deupree y otros, 2002). Estados Unidos y Australia, dos países típicamente exportadores de educación superior, son los patrocinadores de este proceso de liberalización, que puede representar un cambio enorme para el futuro de las instituciones universitarias y para la formación que ellas imparten. En dicho sentido, uno de los cambios más «peligrosos» para la actual estructura de las universidades tradicionales es la posibilidad de que éstas pierdan su 
privilegio nacional de ser expedidoras de títulos académicos (y en consecuencia de los profesionales, tal como ocurre en muchos países). $\mathrm{Si}$ eso sucediera -y es verosímil que antes o después suceda-, las universidades tendrían que competir a un nivel inimaginable en estos momentos.

\subsection{LA SOCIEDAD DEL CONOCIMIENTO}

La aparición de la llamada sociedad del conocimiento es otro de los cambios en el contexto de la educación superior que va a ejercer mayor influencia sobre el funcionamiento de las universidades (CE, 1995, 1997, 2003). El valor económico de la educación, yen particular de la superior, ha estado latente desde el siglo XIX, cuando las universidades se dispusieron a dar respuesta a las nuevas demandas de la era industrial. Sin embargo, fue en la segunda mitad del siglo XX cuando el valor económico de la educación fue universalmente reconocido por los analistas (Mora y Vidal, 2003). Durante ese período, que fue testigo de un gran desarrollo tecnológico, los expertos empezaron a percibir con claridad que ese desarrollo sólo era posible si se disponía de recursos humanos muy cualificados. En la sociedad del conocimiento, a diferencia de la sociedad industrial, se considera que son el conocimiento y la tecnología, y ya no la mera producción industrial, los elementos de mayor impacto para el desarrollo económico y social de las comunidades.

El entorno de las universidades está cambiando en esta sociedad que emerge, con las siguientes características (Scott, 1996):

- Aceleración de la innovación científica y tecnológica.

- Rapidez de los flujos de información en una nueva dimensión del espacio y del tiempo.

- Aumento del riesgo en la mayoría de los fenómenos, de la complejidad, de la no-linealidad y de la circularidad.

En esta sociedad adquieren nueva relevancia la educación superior y las universidades, ya que éstas no sólo son una de las principales fuentes para generar conocimiento (gran parte de la investigación que se realiza en los países la llevan a cabo las universidades), sino que son ellas los centros básicos de transmisión del conocimiento, de la ciencia y de la tecnología. Si las universidades han sido importantes en 
la era industrial, su papel en la sociedad del conocimiento puede ser mucho más importante, siempre que sepan responder con flexibilidad a las nuevas demandas de esa sociedad del conocimiento. La universidad se vislumbra como una generadora de conocimiento, aunque no la única, y como una empresa al servicio de las necesidades de formación y de desarrollo tecnológico del entorno dentro de la sociedad del conocimiento.

\subsection{LA UNIVERSIDAD UNIVERSAL}

En las últimas décadas, las viejas instituciones medievales han experimentado la transformación más importante de toda su historia: pasar de ser unos establecimientos dedicados a formar a las elites, a convertirse en el lugar de formación de una gran parte de la población, lo que ha venido en llamarse un sistema de educación superior universal (Trow, 1974). Las universidades, por primera vez en su trayectoria, se han hecho universales en tres sentidos:

- En su expansión geográfica, ya que se han extendido a todos los lugares, de modo que apenas es necesaria la movilidad física para poder asistir a un centro de educación superior. Pero, además, el desarrollo creciente de la educación virtual (a pesar de las limitaciones que todavía tiene este tipo de educación), permite el acceso a la formación superior desde cualquier lugar y en cualquier situación.

- La educación superior también se está haciendo universal en el sentido temporal. Mientras que por tradición dicha educación había sido algo preparado casi en exclusiva para los jóvenes que finalizaban sus estudios secundarios, en la actualidad existe una tendencia creciente a ofrecer estudios a lo largo de toda la vida para cubrir las necesidades de formación continua de los profesionales, pero también las de una demanda cultural creciente de la población adulta.

- Porúltimo, la universidad se ha hecho universal en el acceso. En todos los países desarrollados, así como en muchos otros en vías de desarrollo, una proporción muy elevada de jóvenes que finalizan sus estudios secundarios accede a la educación superior. 
La universidad del futuro se vislumbra como una institución que suministrará formación a la gran mayoría de la población a lo largo de toda la vida. Una universidad como ésta, extendida a una gran parte de la población, a través de toda su vida, y accesible en todo lugar, no es igual ni puede funcionar del mismo modo que la vieja, todavía muy reciente, a la que sólo ingresaban los pocos jóvenes que disponían de recursos elevados y que provenían de los estratos sociales y culturales más altos de la sociedad. La universidad universal debe plantearse nuevos objetivos y nuevos modos de funcionamiento, que no se corresponden con los que han estado vigentes desde la Edad Media hasta hace muy poco (Mora, 2001).

\subsection{EL ESPACIO EUROPEO DE EDUCACIÓN SUPERIOR}

En Europa, uno los cambios que hay que tener más en cuenta en el contexto de las universidades es el actual proceso de armonización de la educación superior, conocido como proceso de Bolonia. La preocupación por los problemas de la educación superior europea llevó a 29 ministros de educación del continente a firmar la Declaración de Bolonia (1999), que ha sido el punto de partida del importante proceso de renovación en el que hoy están inmersas las universidades europeas.

La Declaración de Bolonia señala que es necesario «desarrollar Europa, fortaleciendo sus dimensiones intelectual, cultural, social, científica y tecnológica». Asimismo, señala que hay que «asegurar que el atractivo de la educación superior europea sea tan alto como el de sus tradiciones culturales». Mejorar el atractivo y la aplicabilidad de la educación superior al desarrollo de la sociedad en el sentido más amplio, son los principios que inspiran la Declaración de Bolonia.

En su desarrollo, dicha Declaración señala que, para el año 2010, deberá haberse establecido el Espacio Europeo de Educación Superior, con el fin de alcanzar estas tres metas:

- Mejorar la competitividad y el atractivo internacional de la educación superior europea.

- Mejorar la empleabilidad de los graduados europeos.

- Desarrollar la movilidad interna y externa de estudiantes y de graduados. 
En el fondo, el proceso de Bolonia es el resultado de dos fuerzas directrices: la necesidad de adaptarse a la sociedad del conocimiento, y la exigencia de acomodarse a un mundo globalizado en el que la europeización sea sólo un primer paso. El llamado proceso de Bolonia es el procedimiento de cambio de la educación superior más importante que ha ocurrido desde principios del siglo XIX, cuando las universidades europeas se adaptaron a la era industrial [Hortale y Mora (en prensa)].

\subsection{UN NUEVO MODELO DE UNIVERSIDAD}

Todos los cambios de contexto que hemos mencionado conducen a la definición de lo que podríamos llamar un nuevo modelo de universidad, caracterizado por la globalización (compitiendo en un entorno global), por la universalidad (sirviendo a todos y en todo momento), y por la necesidad de dar respuesta a las nuevas demandas de la sociedad del conocimiento. El proceso de Bolonia no es nada más que la concreción a nivel europeo de este nuevo cambio de contexto que se extiende mucho más allá. Sin embargo, aunque las tendencias generales parecen claras, el nuevo contexto, precisamente por ser nuevo, es incierto y complejo. Si bien se pueden prever las grandes tendencias, los detalles exigen una actitud de constante reflexión y análisis, con el fin de que las universidades sean capaces de responder con rapidez y solvencia a los cambios de contexto. La universidad no es la misma de antes. Es una nueva institución que debe adoptar nuevos objetivos y acoger mecanismos flexibles de adaptación continua a esos objetivos. Todo un reto para unas instituciones como las europeas, en buena medida lastradas por el conservadurismo.

\section{LA RESPUESTA DE LAEDUCACION SUPERIOR}

Hemos revisado en el apartado anterior al gunos de los aspectos que marcan el cambio de contexto al que se enfrenta la educación superior. La cuestión ahora es tratar de analizar cuál debe ser la respuesta de la educación superior a ese cambio de contexto. En este apartado señalaremos al gunos de los aspectos que, en nuestra opinión, deben ser cambiados en las universidades para dar una contestación adecuada a las demandas de la sociedad. 


\subsection{CAMBIO DEL MODELO EDUCATIVO: DE LA ENSEÑANZA AL APRENDIZAJE}

El mercado laboral de la sociedad del conocimiento es diferente al de la era industrial. Salvo excepciones, las profesiones ya no están tan claramente definidas. La multidisciplinariedad es una necesidad creciente en los supuestos de trabajo. Por otro lado, los conocimientos se convierten en obsoletos en muy breve período de tiempo. Los modelos pedagógicos tradicionales, en los que un profesor trataba de enseñar el estado del arte de una profesión, ya no sirven. Hay que crear un entorno de aprendizaje continuo alrededor de los estudiantes que les capacite para seguir aprendiendo a lo largo de toda la vida, y que les permita permanecer receptivos a los cambios conceptuales, científicos y tecnológicos que vayan apareciendo durante su actividad laboral. Hay que pasar de un modelo basado en la acumulación de conocimientos a otro fundamentado en una actitud permanente y activa de aprendizaje. Dado que la transmisión de conocimientos no puede continuar siendo el único objetivo del proceso educativo, el modelo pedagógico sustentado en el profesor como transmisor de conocimientos debe ser sustituido por otro en el que el alumno se convierta en el agente activo del proceso de aprendizaje, que deberá seguir manteniendo durante toda su vida. La función del profesor será la de dirigir y entrenar al estudiante en ese proceso de aprendizaje. El nuevo modelo de créditos ECTS, que se está implantado en las universidades europeas, no es un simple sistema contable sino que representa (o al menos debería representar) un medio para establecer los nuevos roles de los profesores y de los alumnos.

\subsection{CAMBIOS OBJETIVOS: DE LOS CONOCIMIENTOS A LAS COMPETENCIAS}

Como hemos visto, el modelo pedagógico de la universidad tradicional, sobre todo en el caso español y en el de otros países con un modelo napoleónico, y los conocimientos, en especial los teóricos, son los aspectos a los que mayor importancia concede el sistema educativo superior. Sin embargo, todo indica que las necesidades del nuevo contexto de la educación superior exigen, además de los conocimientos, formar a los individuos en un amplio conjunto de competencias que incluyan por supuesto los conocimientos, pero también las actividades y las actitudes que son requeridas en el puesto de trabajo.

El conjunto de cualificaciones que necesita un trabajador para ocupar con solvencia un puesto laboral, es conocido hoy con la denomi- 
nación de competencias. La definición que los expertos dan a este término es la siguiente: «Una persona tiene competencia ocupacional si posee los conocimientos, las destrezas y las aptitudes que necesita para desenvolverse en una ocupación, si es capaz de resolver tareas independiente y flexiblemente, y si tiene la voluntad y la capacidad de desarrollar su esfera de trabajo dentro de la estructura organizativa en la que está inmerso» (Bunk, 1994, p. 9). El siguiente cuadro, adaptado de Bunk (1994), resume las distintas dimensiones que constituyen el concepto de competencia:

\begin{tabular}{|c|c|c|c|}
\hline $\begin{array}{l}\text { Competencias } \\
\text { especializadas }\end{array}$ & $\begin{array}{l}\text { Competencias } \\
\text { metodológicas }\end{array}$ & $\begin{array}{l}\text { Competencias } \\
\text { sociales }\end{array}$ & $\begin{array}{l}\text { Competencias } \\
\text { participativas }\end{array}$ \\
\hline $\begin{array}{l}\text { - Conocimiento. } \\
\text { - Destrezas. } \\
\text { - Habilidades. }\end{array}$ & - Flexibilidad. & $\begin{array}{l}\text { - Sociabilidad. } \\
\text { - Modos de } \\
\text { conducta. }\end{array}$ & - Participación. \\
\hline $\begin{array}{l}\text { - Interdisciplinarios. } \\
\text { - Específicos. } \\
\text { - Extendidos vertical } \\
\text { y horizontalmente. }\end{array}$ & $\begin{array}{l}\text { - Métodos de } \\
\text { trabajo variables. } \\
\text { - Pensamiento } \\
\text { independiente. } \\
\text { - Adaptabilidad. }\end{array}$ & $\begin{array}{l}\text { - Voluntad de } \\
\text { flexibilidad y } \\
\text { adaptabilidad. } \\
\text { - Actitud positiva } \\
\text { hacia el trabajo. } \\
\text { - Voluntad de } \\
\text { cooperar, de juego } \\
\text { limpio. } \\
\text { - Voluntad de } \\
\text { ayudar, de espíritu } \\
\text { de equipo. }\end{array}$ & $\begin{array}{l}\text { - Capacidad } \\
\text { coordinadora. } \\
\text { - Organizativa. } \\
\text { - De persuasión. } \\
\text { - De toma de } \\
\text { decisiones. } \\
\text { - De asumir } \\
\text { responsabilidades. } \\
\text { - De liderazgo. }\end{array}$ \\
\hline
\end{tabular}

En la encuesta ya citada se instaba también a los graduados europeos a que valoraran las competencias que eran requeridas en sus puestos de trabajo, así como en qué medida habían adquirido dichas competencias, bien durante los estudios, bien durante el tiempo que Ilevaban trabajando. La tabla 3 muestra la valoración que dichos graduados hicieron de las competencias exigidas por sus puestos laborales. Como se puede ver, todas ellas están referidas a actividades y a actitudes como son la capacidad de realizar el trabajo independientemente, la de resolver problemas, la de comunicación oral, la de saber asumir responsabilidades, la de saber administrar el tiempo, la de saber planificar, así como las de tener iniciativa, adaptabilidad y lealtad. Se aprecia con claridad, según las opiniones de los propios graduados, que los puestos laborales necesitan un conjunto de competencias que no son las que tradicionalmente preocupan, ni por tanto las que se enseñan en los sistemas más tradicionales de educación superior, sobre todo en los modelos más profesionalistas como el español. 
TABLA 3

Valoración de las competencias requeridas

(Indicador que toma valores entre 1 y 5 )

\begin{tabular}{|l|c|}
\hline Trabajar independientemente & 4,3 \\
\hline Habilidad para resolver problemas & 4,3 \\
\hline Habilidad en comunicación oral & 4,3 \\
\hline Trabajar bajo presión & 4,3 \\
\hline Asumir responsabilidades, tomar decisiones & 4,2 \\
\hline Trabajar en equipo & 4,2 \\
\hline Administración del tiempo & 4,1 \\
\hline Firmeza, resolución, persistencia & 4,1 \\
\hline Exactitud, atención al detalle & 4,1 \\
\hline Planificación, coordinación y organización & 4,1 \\
\hline Iniciativa & 4,1 \\
\hline Adaptabilidad & 4,1 \\
\hline Involucrarse personalmente en el trabajo & 4,1 \\
\hline Lealtad, honestidad & 4,1 \\
\hline Habilidad en comunicación escrita & 4,1 \\
\hline
\end{tabular}

FUENTE: Elaboración propia con datos de la encuesta CHEERS.

Basada en los datos de la misma encuesta, la tabla 4 muestra las diferencias entre los val ores exi gidos y obtenidos de las competencias, ordenados por la mayor o menor diferencia entre el valor requerido y el adquirido (para resaltar las tendencias más relevantes sólo se han incluido las competencias que aparecen en ambos extremos de una larga lista). Una vez más apreciamos que los déficit en competencias de los graduados universitarios están en los relacionados con habilidades y actitudes, mientras que los graduados manifiestan que existe un exceso de competencias en conocimientos generales y teóricos. Por fortuna, una habilidad tan importante como es la capacidad de aprendizaje parece que está bien asimilada por los graduados. La otra competencia en la que 
los graduados muestran superávit es, curiosamente, el dominio de idiomas extranjeros. $\mathrm{Ni}$ el valor requerido ni el adquirido son muy altos, lo que demuestra que la utilización de idiomas extranjeros en Europa es todavía mucho menor de lo que se supone.

TAB LA 4

Valoración de las competencias más y menos requeridas con la valoración del nivel en que han sido adquiridas y la diferencia entre ambos valores

(Indicador que toma valores entre 1 y 5 )

\begin{tabular}{|l||c|c|c}
\hline & Adquiridas & Requeridas & Diferencia \\
\hline Capacidad de negociación & 2,6 & 3,7 & $-1,1$ \\
\hline Planificación, coordinación y organización & 3,1 & 4,1 & $-1,0$ \\
\hline Asumir responsabilidades, tomar decisiones & 3,4 & 4,2 & $-0,9$ \\
\hline \hline Administración del tiempo & 3,3 & 4,1 & $-0,8$ \\
\hline Trabajar bajo presión & 3,5 & 4,3 & $-0,8$ \\
\hline Razonar en términos económicos & 2,8 & 3,5 & $-0,8$ \\
\hline \hline Liderazgo & 2,8 & 3,6 & $-0,7$ \\
\hline Habilidad para resolver problemas & 3,6 & 4,3 & $-0,7$ \\
\hline Habilidad en comunicación oral & 3,6 & 4,3 & $-0,7$ \\
\hline \hline Amplio conocimiento general & 3,7 & 3,6 & 0,1 \\
\hline Conocimiento teórico del campo especifico & 3,8 & 3,7 & 0,1 \\
\hline Habilidad para aprender & 4,2 & 4,0 & 0,2 \\
\hline Dominio de lenguas extranjeras & 3,1 & 2,9 & 0,2 \\
\hline
\end{tabular}

FUENTE: Elaboración propia con datos de la encuesta CHEERS.

Por último, la tabla 5 muestra los resultados de una regresión ordinaria, en la que la variable dependiente era el salario de los graduados universitarios, y las variables explicativas eran un grupo seleccionado de competencias junto con un cúmulo de variables de control (características personales, del puesto de trabajo, de los estudios, etc.) que garantizaran la validez estadística del análisis. Las competencias que fueron incluidas en el análisis se presentan en esta tabla ordenadas de mayor a menor influencia sobre los salarios. Los resultados confirman una vez más la importancia que el mercado laboral está dando a las habilidades y a las actitudes de los graduados, por encima de la que se da a los 
conocimientos. Según este estudio, las competencias que son más influyentes en el salario son: saber asumir responsabilidades, tener capacidad de planificar y de resolver problemas, poseer aptitud para trabajar bajo presión, y contar con habilidades de comunicación oral. EI resto de variables, incluyendo el conocimiento teórico y metodológico del campo específico en el que se ha formado el graduado, no parece influir en los salarios. Bien es cierto que los conocimientos teóricos y metodológicos quizás sean la condición necesaria para poder haber optado al puesto de trabajo que se ocupa, por lo que tampoco hay que reducir su importancia. El hecho es que no son los conocimientos los que marcan la diferencia en salarios entre los graduados, sino sus actitudes y su predisposición hacia el puesto laboral.

TABLA 5

Significatividad de una selección de competencias en una ecuación de salarios

\begin{tabular}{|l|c}
\hline & t-stud \\
\hline Asumir responsabilidades, tomar decisiones & 6,63 \\
\hline Planificación, coordinación y organización & 4,36 \\
\hline Habilidad para resolver problemas & 4,02 \\
\hline Trabajar bajo presión & 2,14 \\
\hline Habilidad en comunicación oral & 2,14 \\
\hline Trabajo en equipo & 1,62 \\
\hline Iniciativa & 1,22 \\
\hline Pensamiento crítico & 1,01 \\
\hline Habilidad en comunicación escrita & 0,84 \\
\hline Conocimiento metodológico en el campo especifico & 0,49 \\
\hline Conocimiento teórico del campo especifico & $-0,90$
\end{tabular}

FUENTE: Elaboración propia con datos de la encuesta CHEERS.

Todos estos resultados muestran que los sistemas de educación superior de al gún modo deberán tratar de formar a los graduados en esas actitudes y habilidades que demanda la sociedad. No es posible sostener 
por más tiempo que los sistemas de educación superior sólo sigan centrados en la formación de conocimientos, sobre todo de conocimientos teóricos, cuando las demandas de la sociedad del conocimiento y del mercado laboral en el que van a trabajar los graduados exige también la formación en otro grupo más amplio de competencias (Valle, 2000; CINDA, 2004). Es necesario transmitir los valores del mercado laboral, aunque, dada la gran diversidad de empleadores (públicos y privados, y, dentro de estos, de múltiples tipologías), tales valores han de ser también generalistas (Kogan y Brennan, 1993). Hay que transmitir también los valores generales relacionados con la cultura del trabajo: mayor atención al entorno laboral, más énfasis en los nuevos estilos de gestión, y mayor importancia a los aspectos culturales y humanos del proceso productivo (OCDE, 1992).

La cuestión es cómo modificar los métodos de enseñanza para poder transmitir esos objetivos. Desde el punto de vista de nuestro análisis, los métodos de enseñanza pueden clasificarse en dos tipos: reactivos y proactivos. En los primeros el profesor actúa y el alumno responde; en los segundos es el alumno el que actúa, mientras que el profesor es ante todo un guía. Los primeros (clases teóricas y prácticas, incluso laboratorios con prácticas dirigidas) permiten suministrar conocimientos e incluso destrezas, pero no competencias metodológicas, sociales o participativas. Para formar en los segundos se necesitan mecanismos educativos distintos: seminarios, aprendizaje interactivo, técnicas de discusión y de presentaciones, técnicas de tomas de decisiones, períodos de prácticas en empresas, etc. Es preciso introducir métodos proactivos que transmitan las competencias que van a necesitar los futuros trabajadores (Mora, 1997). Este es, en esencia, el objetivo que señala la Declaración de Bolonia cuando pide a la educación superior europea que sea capaz de mejorar su empleabilidad. En estos momentos, una educación activa que desarrolle las potencialidades individuales y sociales que el alumno va a necesitar en el futuro debería ser el principal objetivo pedagógico de las universidades y de los enseñantes españoles y europeos.

Conviene resaltar una cuestión importante, que, si bien en apariencia resulta simple, produce más confusión de lo que parece. La formación teórica es un concepto antagónico al de formación práctica, mientras que la formación general es antagónica con la formación especializada. Da la impresión de que con frecuencia se confunde la formación práctica con la formación especializada. La necesidad de desarrollar mucho más la formación práctica en los planes de estudio no 
debe llevar aparejado el desarrollo de la formación especializada; antes al contrario, la formación en competencias (entendida en un sentido amplio) exige impulsar mucho más la formación general que la específica. Por supuesto, una formación general que a la vez sea una formación práctica, en la que los estudiantes tengan la oportunidad no sólo de saber muchas cosas sino de aplicarlas.

Por último, ha sido constatado (Teichler y Kehm, 1995) que en multitud de casos los logros de los estudiantes, tras obtener su grado, están más relacionados con las actitudes y con las acciones de los propios compañeros que con el modo en el que se provee la educación (programas, estilos docentes, etc.). De hecho, la tabla 2 mostraba que la relación con los compañeros era uno de los efectos más valorados por los graduados en su experiencia universitaria. Este resultado obliga, como algo importante, a que las instituciones tomen en consideración la organización de todo tipo de actividades formativas que promuevan los intercambios entre estudiantes, y de éstos con su entorno social y laboral.

\subsection{CAMBIO EN LOS MODELOS ORGANIZATIVOS}

Cuando la mitad de los jóvenes asiste a instituciones de educación superior, no puede seguir pensándose en la universidad como en la torre de marfil en la que se conserva el saber para unos pocos y que se organiza como lo hacía en la Edad Media. Aunque tal vez todos reconocemos este hecho, algunos comportamientos parecen indicar que no todos han acabado de adaptarse a esa realidad. Si la universidad es un lugar de formación para una proporción mayoritaria de la sociedad, su principal deber como servicio público será el de estar atenta a las necesidades globales de esa sociedad, que son muy distintas de las necesidades tradicionales de las elites o de las del propio Estado. Eso exige nuevos modelos organizativos bastante más flexibles y ágiles.

El cambio en la misión de la universidad, la necesidad de mejora, la creciente complejidad de las instituciones de educación superior, la competitividad y la diversificación a nivel internacional y nacional, hacen que una mayor inclinación de los sistemas universitarios hacia la sociedad pueda ser un medio poderoso de estimular la sensibilidad de las instituciones para satisfacer las demandas de esa sociedad (M eek y otros, 1996; N eave y van Vught, 1991). Esta «marketization» de la educación superior (Williams, 1995) está basada en la creencia de que la introducción de tendencias de mercado en la educación superior 
proporcionará incentivos a las universidades para mejorar la calidad de la enseñanza y de la investigación, para impulsar la productividad académica, para estimular la innovación en los programas académicos, y para avanzar en los servicios que proporciona a la sociedad en general (Dill, 1997). La sociedad del conocimiento exige a la universidad convertirse en una empresa de servicios múltiples, en algo que al guien ya se ha atrevido a calificar como broker del conocimiento y de las competencias profesionales (Monasta, 1997).

La universidad ha dejado de ser el lugar de formación de las elites dirigentes del Estado y de las grandes empresas. Por otro lado, aquella ya no es un centro en donde se puede cultivar sólo la alta investigación. Cada vez más se le exige que sea el motor de la investigación aplicada y del desarrollo tecnológico de la comunidad. Este es un hecho generalizado, y las universidades más dinámicas del mundo son hoy centros de I+D profundamente imbricados en su entorno (Clark, 1998). A pesar de lo anterior, la universidad debe mantener la formación de elites, la investigación científica pura y el análisis critico de la sociedad. No cultivar estos aspectos seria impedir el desarrollo futuro de la propia institución y de la sociedad.

La no-diferenciación de las instituciones de educación superior, como es el caso español y el de la mayoría de los países latinoamericanos, hace que ambos tipos de misiones (la formación de masas y la de elites científicas) estén encomendadas a la universidad. Las universidades deben buscar un equilibrio razonable entre los dos objetivos. Eso exige un proceso de diferenciación, tanto entre universidades como dentro de las propias instituciones, y tanto en el aspecto docente como en el investigador. Las universidades deben buscar su mejor nicho para el desarrollo de sus potencialidades, que no es imprescindible que sea el de la excelencia científica o el de la calidad investigadora. Seria razonable que una mayor proporción de los programas de estudios de las universidades hiciera hincapié en la calidad de la enseñanza como meta casi exclusiva.

Las diferencias entre instituciones, y dentro de ellas, deberían ser explícitas a través de la publicación de los objetivos de los distintos programas educativos y de la información externa que pueden proporcionar los mecanismos de evaluación y de acreditación actualmente en marcha. Así, los alumnos tendrían oportunidad de el egir entre programas de acuerdo con criterios distintos de la mera proximidad geográfica. Esto produciría mejoras en la eficiencia generadas por la competencia entre 
los alumnos más aventajados, por la mejora del prestigio de las instituciones, y, en consecuencia, por la obtención de los recursos financieros ligados a resultados.

Otro aspecto que requiere cambios del modelo organizativo es el de la temporalización del proceso de aprendizaje. El sistema educativo superior ha estado tradicionalmente enfocado a atender a jóvenes estudiantes cuando finalizaban sus estudios secundarios. En estos momentos en los que la sociedad del conocimiento exige la formación continua de todos los que están inmersos en el proceso productivo, la preparación que proporcionan las universidades ya no puede estar ni exclusiva ni fundamentalmente enfocada a la formación de los jóvenes, sino a extenderla a todos aquellos que quieran aprovechar sus enseñanzas a lo largo de sus vidas. Llevar adelante este cambio supone modificaciones profundas del sistema organizativo, permitiendo vías mucho más flexibles entre los distintos estudios, entre los diferentes programas y entre la universidad y el mercado laboral. La multidisciplinariedad y la intercomunicación de los programas educativos es una necesidad que debe plantearse dentro de los nuevos modelos organizativos de las instituciones de educación superior.

\subsection{UN NUEVO ENFOQUE PARA LOS PROCESOS DE EVALUACIÓN}

Los sistemas de evaluación de cualquier proceso tienen un gran impacto sobre el desarrollo de ese mismo proceso. En el caso de la educación, es un hecho evidente que el modo de evaluar hace que los sistemas se adapten más rápido y mejor a los objetivos que ha marcado previamente el modelo de evaluación. En las últimas dos décadas se han desarrollado en Europa y en Latinoamérica procesos de evaluación de la educación superior basados casi siempre en el análisis de los procesos. La hipótesis subyacente consistía en que unos procesos adecuados deberían producir unos resultados también correctos. Sin embargo, la experiencia acumulada y los nuevos objetivos de la educación universitaria nos inducen a proponer nuevos modelos de evaluación que estén más enfocados a la evaluación de los resultados que a la de los procesos. La razón de este cambio es consecuente por las ideas que hemos ido presentando. El nuevo modelo educativo deberá estar centrado en el aprendizaje; por tanto, más en los resultados de ese aprendizaje que en cómo se ha realizado el proceso. Si el objetivo del nuevo modelo educativo tuviera que ser la formación en competencias, lo importante sería valorar si tales competencias han sido adquiridas por los estudian- 
tes, y no tanto el modo en el que han sido adquiridas. Una evaluación centrada en los procesos, como ha sucedido hasta ahora, sería de algún modo incompatible con la flexibilidad y con la diferenciación que propugnamos para el nuevo modelo educativo.

De lo que se trataría, por tanto, sería de evaluar en qué medida los grandes objetivos de formación en competencia son alcanzados por las instituciones de educación superior. Este enfoque es el que debe estar detrás de los procesos de acreditación que actual mente se están implantando en Europa. De hecho, el modelo holandés de acreditación y el proyecto de modelo español tienen este enfoque, centrado en la evaluación de los resultados. Recientes experiencias latinoamericanas van en el mismo sentido (CINDA, 2004), así como los criterios que se están discutiendo para iniciar los primeros pasos en la acreditación de titulaciones, que son los que recoge el documento elaborado por el grupo de trabajo de la J oint Quality Initiative sobre descriptores compartidos para definir las características de una titulación (J Ql, 2003). Las futuras evaluaciones y acreditaciones de los programas tienen que estar orientadas a valorar en qué medida son alcanzados los objetivos de formación en competencias. Eso exige la definición de nuevos instrumentos evaluadores, que, además, ayuden a las universidades a transformar sus objetivos pedagógicos en el mismo sentido.

\section{CONCLUSIONES}

El cambio de contexto para la educación superior (sociedad global, sociedad del conocimiento, universalidad, etc.) exige realizar cambios en el sistema educativo superior para dar respuesta a los nuevos retos planteados. Los cambios que hay que realizar son de dos tipos: intrínsecos (del modelo pedagógico) y extrínsecos (del modelo organizativo de las instituciones):

- La idea esencial del cambio intrínseco se puede sintetizar en la necesidad de cambiar el paradigma educativo desde un modelo basado casi con exclusividad en el conocimiento, a otro sustentado en la formación integral de los individuos. Es necesario que los sistemas de educación superior dediquen una atención especial al desarrollo de las habilidades metodológicas (en esencia, los viejos principios de «saber leer», «saber hablar y escribir», «saber pensar» y «saber 
seguir aprendiendo autónomamente»), de las sociales y participativas (aprender a relacionarse y a entender el mundo del trabajo), y también a desarrollar los conocimientos de carácter práctico que faciliten la aplicación de los conocimientos teóricos.

- El cambio extrínseco, es decir, el del modelo organizativo de las instituciones de educación superior, debe estar orientado al aumento de la flexibilidad del sistema en un sentido temporal (facilitando la formación a lo largo de la vida) y operativo (facilitando el paso del sistema educativo al mercado laboral, y entre programas dentro del sistema educativo).

En esencia, el cambio se reduce a abrir las puertas a la sociedad y a escuchar lo que ésta demanda de las universidades. Eso exige una actitud de servicio social de las instituciones, y, sobre todo, de cada uno de sus miembros, en especial de los docentes que han de aplicar estos cambios.

\section{BIBLIOGRAFÍA}

BUNK, G. P. (1994): «Teaching Competence in Initial and Continuing Vocational Training in the Federal Republic of Germany», en Vocational Training European J ournal, 1 , pp. 8-14.

CLARK, B. R. (1998): Creating Entrepreneurial Universities, Pergamon.

COMISIÓN EUROPEA (1995): White Paper on Education and Training: Teaching and Learning; Towards the Learning Society, Documento Com (95) 590. (97) 563.

COMISIÓN EUROPEA (1997): Towards a Europe of Knowledge, Documento COM

COMISIÓN EUROPEA (2003): The Role of the Universities in the Knowledge Society. http://europa.eu.int/eur-lex/es/com/cnc/2003/com2003_0058es01.pdf.

CINDA (2004): Competencias de egresados universitarios, Santiago de Chile.

DECLARACIÓN DE B OLONIA(1999):Documento disponible en http://europa.eu.int/ comm/education/policies/educ/bologna/bologna.html. 
DeUPREE, J . L.; J OHNSON, M. E. y LENN, M.P. ( eds.) (2002): OCDE- US, Forum on Trade in Educational Services: Conference Proceedings, Washington, The Center for Quality Assurance in Higher Education.

DILL, D. D. (1997): «Higher Education Markets and Public Policy», Higher Education Policy, 10, 3/4, pp. 167-185.

HORTALE, V. y MORA, J. G.: «Tendências das reformas da Educação Superior na Europa no contexto do processo de Bolonia», en Educação \& Sociedade (en prensa).

J QI (2003): http://www.jointquality.org.

KogAn, M. y BRENNAN, J . (1993): «Higher Education and the World of Work: An Overview», Higher Education in Europe. XVIII, 2, pp. 2-23.

Meek, L.; Goedegebuure, L.; Kivinen, O. y Rinne, R. (1996):The Mockers and Mocked: Comparative Perspectives on Differentiation, Convergence and Diversityin Higher Education, Pergamon.

MONASTA, A. (1997): «Higher Education as the Producer, Transmitter, and B roker of Knowledge as well as Competence», en Higher Education in Europe, XXII, 3, pp. 293- 302.

MORA, J. G.: «Higher Education in Spain: Searching for a Model», en P. Altbach y J. Forest (eds.): International Handbook of Higher Education, Kluwer Pub. (en prensa).

- (2001): «Governance and Management in the New U niversity», en Tertiary Education and Management, 7, pp. 95-110.

- (1997): «Cualificación y empleo tras la educación postobligatoria», en ICE, Revista de Economía, 764, pp. 129-145.

MORA, J . G. yVILA, L. E . (2003): «The Economics of Higher Education», en Roddy Begg (eds.): en The Dialogue Between Higher Education Research and Practice, Kluwer Pub, pp. 121-134.

Neave, G. y van Vught, F. (1991): Prometheus Bound, Pergamon.

OCDE (1992): From Higher Education to Employment, París, OCDE.

S CoTT, P. (1996): «U niversity Governance and Management. An Analysis of the System and Institutional Level Changes in Western Europe», en P. Maassen y F. van Vught (eds.): Inside Academia, Utrecht, de Tidjstroom.

SPORN, B. (1996): «Managing University Culture: an Analysis of the Relationship Between Institutional Culture and Management Approaches», en HigherEducation, 32, pp. 41-61.

TEICHLER, U. y KEHM, B. M. (1995): «Towards a new Understanding of the Relationships Between Higher Education and Employment», en European Journal of Education, 30, 2, pp. 115-132.

TeICHLER, U. y SChOnbuRg, H. (eds.) (2004): Comparative Perspectives on Higher Education and Graduate Employment and Work - Experiences from Twelve Countries, Kluwer Pub. 
México, UNAM.

VALLE, M. A. (2000): Formación en competencias y certificación profesional,

Trow, M. (1974): «Problems in the Transition from Elite to mass Higher Education», en Policies for Higher Education, OCDE.

WILLIAMS, G. (1995): «The "Marketization" of Higher Education: Reforms and Potential Reforms in Higher Education Finance», en D. Dill y B. Sporn (eds.): Emerging Patterns of Social Demand and University Reform: Through a Glass Darkly, Pergamon. 


\title{
Contactar
}

Revista lberoamericana de Educación

\author{
Principal OEI
}

